\title{
Estudios y perspectivas sobre ecología vegetal en México
}

\author{
MIGUEL MARTÍNEZ-RAMOS \\ Centro de Ecología, UNAM. Apdo Postal 70-275, Coyoacán 04510, México, D.F.
}

\begin{abstract}
Resumen. Una revisión histórica de los artículos publicados por autores mexicanos en el campo de ecología vegetal muestra un crecimiento vigoroso de esta disciplina en México. La investigación se ha llevado a cabo principalmente en tres tipos de vegetación (bosque tropical perennifolio, bosque tropical caducifolio y matorral xerófilo), otros tienden a cobrar interés (por ejemplo, bosque mesófilo de montaña y bosques de coníferas y encinos) pero otros han sido pobremente estudiados (por ejemplo, vegetación costera, de dunas y vegetación acuática). La mayoría de los estudios publicados han abordado temas de ecología de comunidades, ecología fisiológica y ecología de poblaciones. En estas áreas de estudio, se ha generado conocimiento de relevancia universal. En México se desarrolla una académica sólida de investigadores y docentes, la cual ha generado un proceso importante de formación de recursos humanos en el área de ecología vegetal. La actividad de los grupos de investigación mexicanos, usando como sistemas de estudio al rico banco de plantas que existen en el país, augura el florecimiento de una escuela importante de esta disciplina en México. Sin embargo, la maduración de esta escuela depende, entre otras cosas, de la organización de instituciones y grupos de investigación y docencia (por ejemplo, definiendo planes, programas y protocolos comunes e interdisciplinarios) y del uso coordinado y racional de los recursos académicos y físicos existentes.
\end{abstract}

Abstract. A historical review of the papers published by Mexican authors in the field of plant ecology shows the notorious growth of this field in Mexico. The research so far undertaken has focused mainly in three types of vegetation (tropical rain forests, tropical deciduous forests and xerophytic shrubs); others are becoming of increasing interest (i.e., tropical cloud forests, temperate oak and pine forests), but some others have been poorly studied (i.e., aquatic and coastal vegetation). Most of the published studies are on subjects such as community ecology, ecophysiology and population ecology. The generated knowledge is of universal relevance. A solid academy of researchers and teachers is developing in Mexico, process that has led to the formation of new human resources in the field of plant ecology. The activity of the Mexican research teams that have as study object the rich vegetation present in the country, promises the emergence of an important school in plant ecology in Mexico. However, the fulfillment of this emergence depends, among some other reasons, on the appropriate organization of institutions and research and teaching teams (i.e., defining plans, programs and protocols) and on the rational and coordinated use of physical and academic resources existing at present.

\section{INTRODUCCIÓN}

Pocos países del mundo poseen un reservorio vegetal tan diverso como el que existe en México. En este país es posible encontrar a casi todos los tipos de vegetación existentes en el planeta y a la décima parte del total de las especies de plantas vasculares (ca., 248,000 sensu Wilson, 1992), hasta ahora registradas a nivel global (Rzedowski, 1978, 1991). Tal diversidad se despliega a través de una gran variedad de ambientes. Algunas de las razones que explican la presencia de este banco enorme de plantas se relacionan con la notable heterogeneidad orográfica, hidrográfica, de suelos y climática del país, con la rica historia biogeográfica de la flora (Rzedowski, 1991; Wendt, 1991) y con la existencia de una gran diversidad faunística que coexiste con dicho complejo vegetal.

Heredera de una fuerte tradición botánica (Sarukhán, 1981; Guevara, 1990; Dirzo, 1993), la ecología vegetal en México ha experimentado un notable crecimiento durante la última década. Más que nunca, se encuentran activas la investigación y el proceso de formación de recursos humanos en esta área científica (Moreno-Casasola y Sánchez, 1990; Moreno-Casasola, este número).
En este trabajo se revisa la situación de la ecología vegetal en México y algunas de sus posibles perspectivas de desarrollo. Mi revisión parte en la década de los ochentas y para cubrir mis objetivos, utilizaré los artículos publicados en revistas de circulación nacional e internacional y libros de relevancia.

Este ensayo consta de dos secciones. En la primera, presento un análisis de la actividad de investigación que se ha llevado a cabo en estas áreas durante los últimos 15 años (1980-1994). Para este análisis usaré los artículos publicados en revistas científicas de amplia difusión, realizados por colegas que llevan a cabo su investigación en instituciones mexicanas. En la segunda sección propongo algunas de las posibles perspectivas de crecimiento de la ecología vegetal en México y sus posibles implicaciones para el conocimiento universal y su aplicación en beneficio de la sociedad.

\section{PRODUCCIÓN CIENTÍFICA SOBRE ECOLOGÍA VEGETAL EN MÉXICO}

Dirzo (1993) analizó la actividad científica realizada por los grupos de investigación mexicanos en el campo de la eco- 
logía vegetal hasta antes de 1989 . No obstante la notable actividad de este campo, que ya se daba hacia finales de la década pasada, Dirzo destacó la reducida cantidad de artículos científicos publicados en revistas de amplia circulación. Por ejemplo, encontró que en el Boletín de la Sociedad Botánica de México se publicaron, durante el periodo de 1944 (año en el que apareció el primer número de esta revista) a 1987, sólo 52 (de un total de 325 ) artículos que abordaron temas ecológicos. En revistas foráneas de circulación internacional la contribución hasta 1989 había sido aún más reducida. Por ejemplo, ocho artículos en un total de 77 volúmenes del Journal of Ecology, diez en Vegetatio (durante el periodo de 1948-1989), 19 en Biotropica a lo largo de sus 29 años de existencia.

Esta situación ha ido mejorando, notablemente durante los últimos cinco años. En la Figura 1 se muestra una estimación del ritmo de crecimiento que se ha dado en el número de publicaciones de artículos científicos de los ecólogos mexicanos, que trabajan con sistemas vegetales, a lo largo de los últimos 45 años. Durante los últimos cinco (1990-1994) el crecimiento se ha acelerado a un ritmo de 25 artículos por año, que es cuatro veces mayor que aquél de una década atrás. Tal patrón temporal de producción sugiere que el campo de ecología vegetal en México es muy dinámico, joven y que actualmente se encuentra en una fase de crecimiento vigoroso.

Obviamente, este crecimiento se debió a que el número de grupos de investigación aumentó a través del tiempo. En el Cuadro 1, se muestra que en México existen cuando menos 11 instituciones que producen de manera regular información científica en el campo de ecología vegetal, en una o varias de sus áreas de estudio. Sin embargo, el crecimiento se debió también a que los productos de la investigación finalizan en la actualidad con mucho más frecuencia en la publicación formal. Para evaluar esta apre- ciación, puede compararse el número de trabajos de ecología vegetal presentados en los congresos nacionales organizados por la Sociedad Botánica de México durante dos periodos del proceso de crecimiento mostrado en la Figura 1. Durante los Congresos I al VII (que abarcaron el período de 1960 a 1980) se presentaron 2,427 contribuciones de las cuales $29 \%$ (704) correspondieron a temas ecológicos, equivalente a un promedio de 35.2 trabajos presentados por año (Dirzo, 1993). Durante los cinco congresos posteriores (1980 a 1993) se presentaron 3,245 trabajos (entre ponencias, carteles y simposios) de los cuales $25.6 \%$ (833) abordaron temas ecológicos, es decir, un promedio de 61.1 trabajos por año. Estos números indican que durante los últimos quince años virtualmente se duplicó la cantidad de trabajos presentados en congresos. Sin embargo, esta relación es tres veces menor a la proporción con la que aumentó la producción de artículos publicados, la cual creció (ver Fig. 1) entre los mismos periodos 7.4 veces. Por lo tanto, le eficiencia de publicación ha aumentado de manera importante.

La Figura 1 también resalta la amplitud con la que se difunde el trabajo de los ecólogos mexicanos. El porcentaje de los artículos que se publicaron en revistas extranjeras de circulación internacional ha aumentado de un $40 \%$ (en el lustro 1980-1984) a alrededor del 75\% (en el lustro 19901994; Fig. 1); es decir, aproximadamente tres de cada cuatro artículos se publican actualmente en revistas foráneas de circulación global.

Las revistas nacionales que con más frecuencia se han usado como medios de publicación son tres: la revista de la Sociedad Botánica de México, la revista Biótica (que publicaba el desaparecido Instituto Nacional de Recursos Bióticos) y la revista Acta Botánica Mexicana (que es editada por el Instituto de Ecología, A.C.; Fig. 2a). En la muestra analizada, encontré que se han publicado artículos en 81 revistas extranjeras. Revistas que tiene un fuerte

CuAdro 1. Algunas de las instituciones principales donde se llevan a cabo estudios en las diferentes áreas de la ecología vegetal en México. $\mathrm{EF}$ = ecofisiología, $\mathrm{EM}$ = ecomorfología, $\mathrm{EP}=$ ecología de poblaciones, $\mathrm{EE}=$ Ecología evolutiva, $\mathrm{EC}=$ ecología de comunidades, $\mathrm{ES}=$ ecosistemas, $\mathrm{ECM}=$ ecología de la conservación y manejo de recursos, $\mathrm{EPj}$ = ecología del paisaje, $\mathrm{EHE}$ = ecología humana y etnoecología.

\begin{tabular}{|c|c|c|c|c|c|c|c|c|c|}
\hline Institución & $\mathrm{EF}$ & EM & EP & $\mathrm{EE}$ & $\mathrm{EC}$ & ES & ECM & $\mathrm{EPj}$ & EHE \\
\hline Centro de Ecología, UNAM & $\mathrm{x}$ & $\mathrm{X}$ & $\mathrm{x}$ & $\mathrm{X}$ & $\mathrm{x}$ & $\mathrm{X}$ & $\mathrm{X}$ & $\mathrm{x}$ & $\mathrm{x}$ \\
\hline Facultad de Ciencias, UNAM & $\mathrm{X}$ & & $\mathrm{X}$ & $\mathrm{X}$ & $\mathrm{x}$ & $\mathrm{X}$ & & $\mathrm{x}$ & \\
\hline Instituto de Biología, UNAM & & $\mathrm{x}$ & & & $\mathrm{X}$ & & & & $\mathrm{X}$ \\
\hline Instituto de Ecología, A.C. & $\mathrm{x}$ & $\mathrm{X}$ & $\mathrm{x}$ & & $\mathrm{X}$ & & $\mathrm{X}$ & $\mathrm{X}$ & $\mathrm{X}$ \\
\hline Centro de Investigaciones Biológicas, CIB & $\mathrm{X}$ & & $\mathrm{x}$ & & $\mathrm{X}$ & & $\mathrm{X}$ & & \\
\hline Centro de Investigaciones del Sureste & & & & & $\mathrm{x}$ & & $\mathrm{X}$ & & \\
\hline Colegio de Posgraduados & & & & & $\mathrm{X}$ & & & & \\
\hline Universidad Autónoma de Sonora & & & $\mathrm{X}$ & & $\mathrm{X}$ & & & & \\
\hline Universidad Autónoma de Yucatán & & & $\mathrm{x}$ & & $\mathrm{x}$ & & & & \\
\hline Universidad Autónoma Metropolitana & & $\mathrm{X}$ & $\mathrm{X}$ & $\mathrm{x}$ & $\mathrm{X}$ & & & & \\
\hline Universidad de Guadalajara & & & & & $\mathrm{x}$ & & & & \\
\hline
\end{tabular}




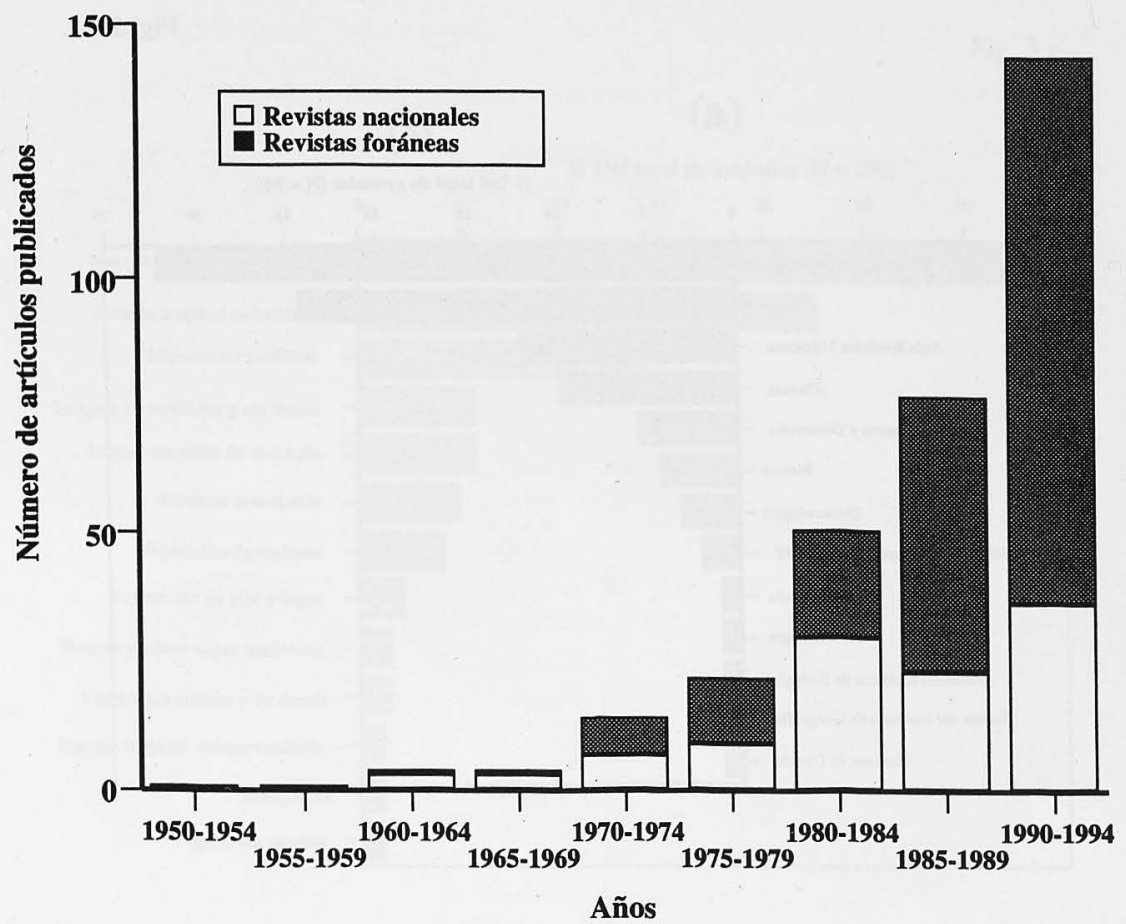

Figura 1. Crecimiento en la producción de artículos científicos en el campo de estudio de ecología vegetal en México (ver texto para detalles).

impacto de difusión en el campo de la ecología, tales como Ecology, Evolution, Oecologia, Journal of Ecology, American Journal of Botany, Oikos, Vegetatio y Journal of Vegetation Science se encuentran entre las 15 revistas foráneas en las que se publica con más frecuencia (Fig. 2b). Todos estos aspectos claramente muestran el esfuerzo que se ha realizado en México para que los resultados de las investigaciones de ecología vegetal tengan una difusión escrita y que esta difusión alcance un ámbito de distribución global en revistas de elevado impacto científico.

\section{PRODUCCIÓN POR TIPOS DE VEGETACIÓN}

Los ecólogos mexicanos han concentrado su actividad principalmente en tres tipos de sistemas vegetales (sensu Rzedowski, 1978): los bosques tropicales perennifolios, los bosques tropicales caducifolios y matorrales xerófilos (Fig. $3 a$ ). En conjunto, los artículos publicados con temas relacionados con estos tres sistemas abarcan casi el $70 \%$ del total de artículos que aparecieron durante el período 1980-1994. Es de llamar la atención que los sistemas de bosque de coníferas y encinares, mesófilos de montaña, de vegetación acuática, manglares, vegetación costera y de dunas han recibido una escasa atención (figura $3 a$ ), no obstante que algunos de estos sistemas tienen una extensión territorial importante en nuestro país. Por ejemplo, en los ochentas se estimaba que los bosques de coníferas cubrían una extensión equivalente al $15 \%$ del territorio mexicano, mientras que los bosques tropicales perennifolios cubrían menos de la mitad de ese extensión (Rzedowski, 1978; Flores y Gerez, 1988).

La preponderancia de artículos sobre ecología de bosques tropicales quizá responde a la fascinación que produce el estudio de la diversidad biológica. También se debe a que en estos sistemas boscosos se encuentran las estaciones biológicas de campo con la mayor tradición de investigación ecológica en México: la Estación de Biología Tropical Los Tuxtlas, Veracruz, y la Estación Experimental Chamela, Jalisco, ambas a cargo de la Universidad Nacional Autónoma de México. Parte de los estudios realizados en Los Tuxtlas se han sintetizado en dos volúmenes editados por el Instituto Nacional de Recursos Bióticos (GómezPompa et al., 1976; Gómez-Pompa y del Amo, 1985), los cuales han tenido un importante impacto internacional. La vegetación de matorral xerófilo, por otro lado, se ha convertido en otro foco de gran actividad de investigación. El volumen recién publicado sobre la ecología del matorral xerófilo, que es protegido por la reserva de El Pedregal de San Angel, UNAM, es muestra de tal actividad (Rojo, 1994). El estudio de este tipo de vegetación es fundamental para México dado que un $35 \%$ de su territorio se encuentra cubierto por matorrales xerófilos (sensu Flores y Gerez, 1988). 
Fig. 2 a

(a)

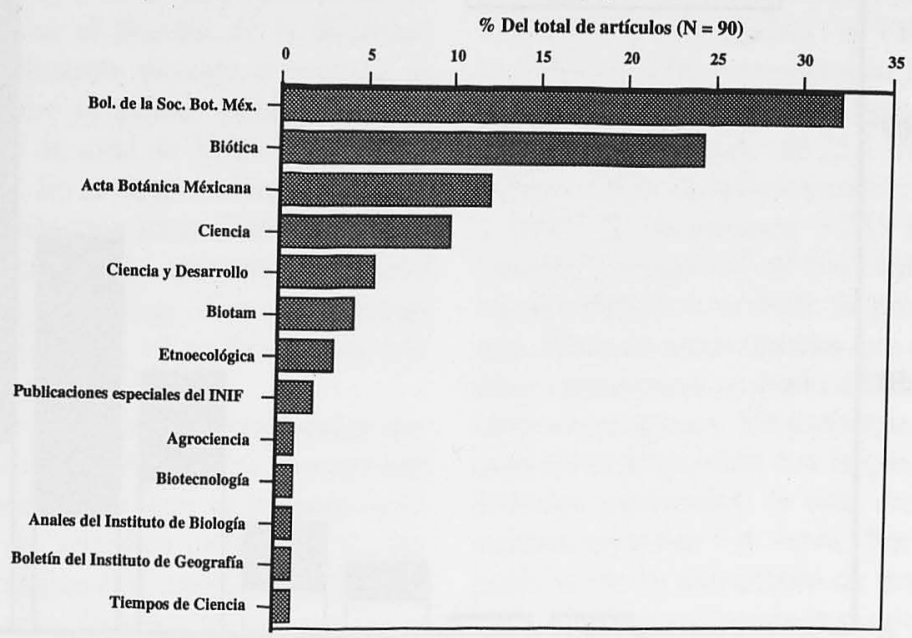

Fig. 2 b

(b)

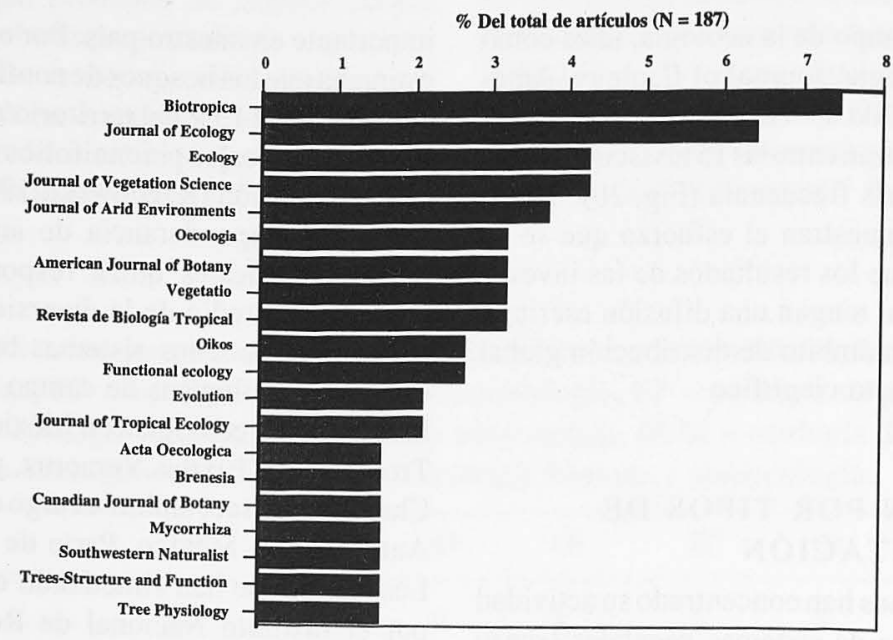

Figura 2. Distribución de frecuencias de los artículos científicos publicados durante el periodo 1980-1994 en el campo de ecología vegetal en México, en (a) revistas nacionales y (b) extranjeras de amplia circulación. 
Fig. 3 a

(a)

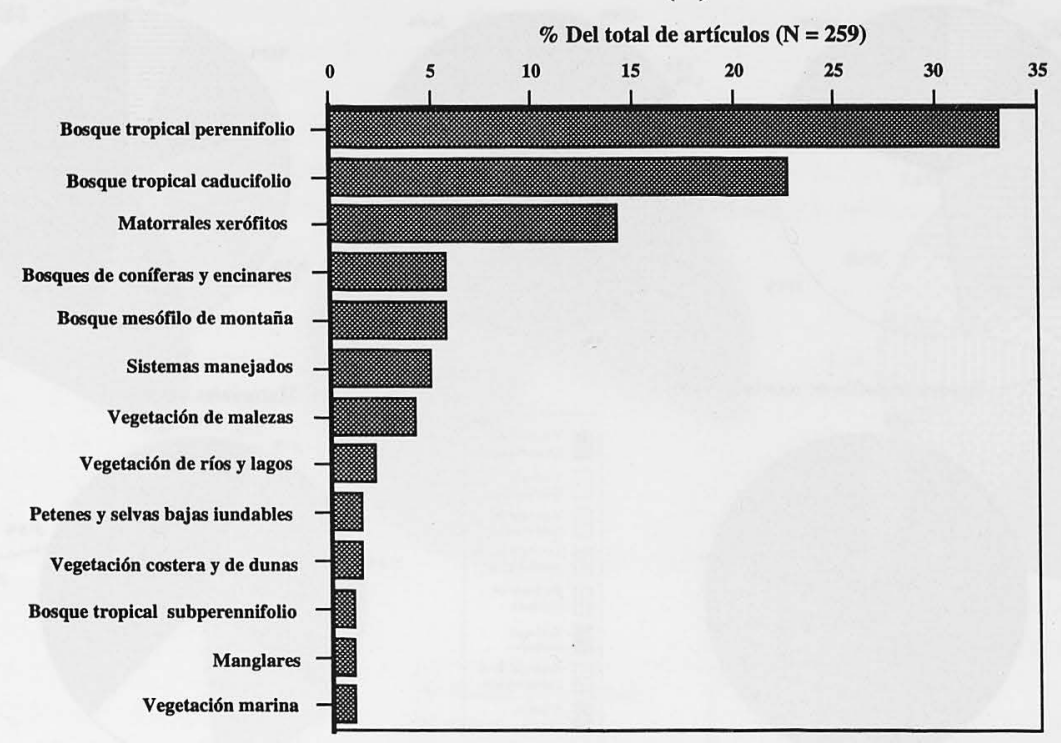

Fig. 3b

(b)

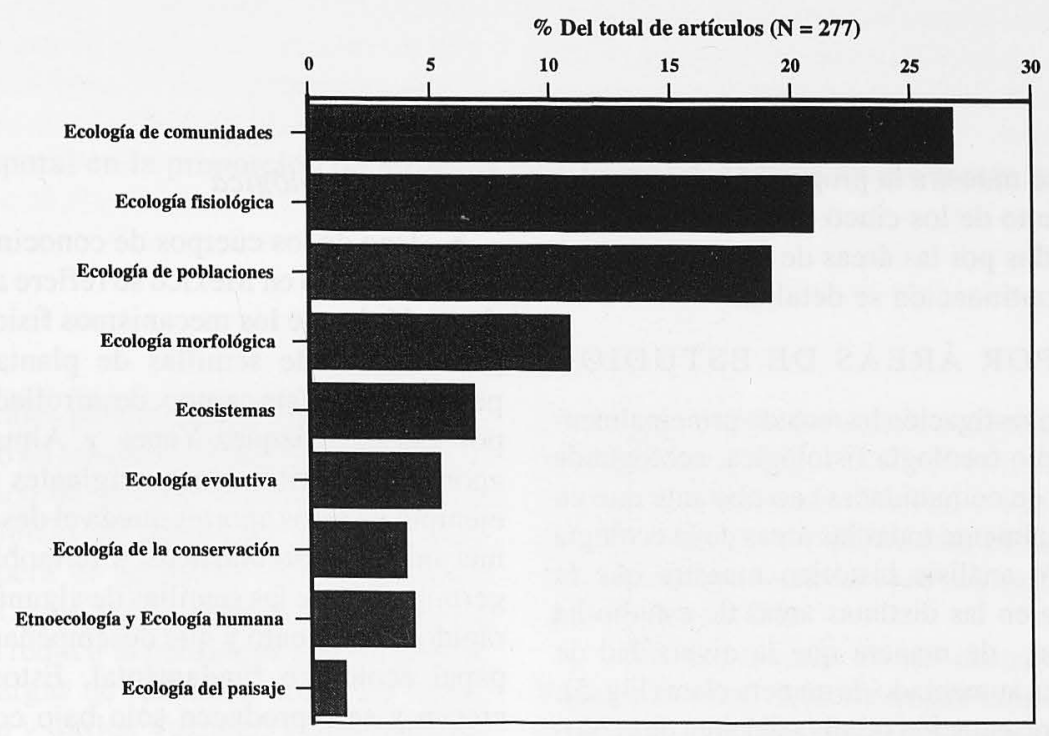

FiguRa 3. Distribución de frecuencias de los artículos científicos publicados durante el periodo 1980-1994 en el campo de ecología vegetal en México, categorizados por temas sobre (a) distintos tipos de vegetación y (b) diferentes áreas de estudios de la ecología. 

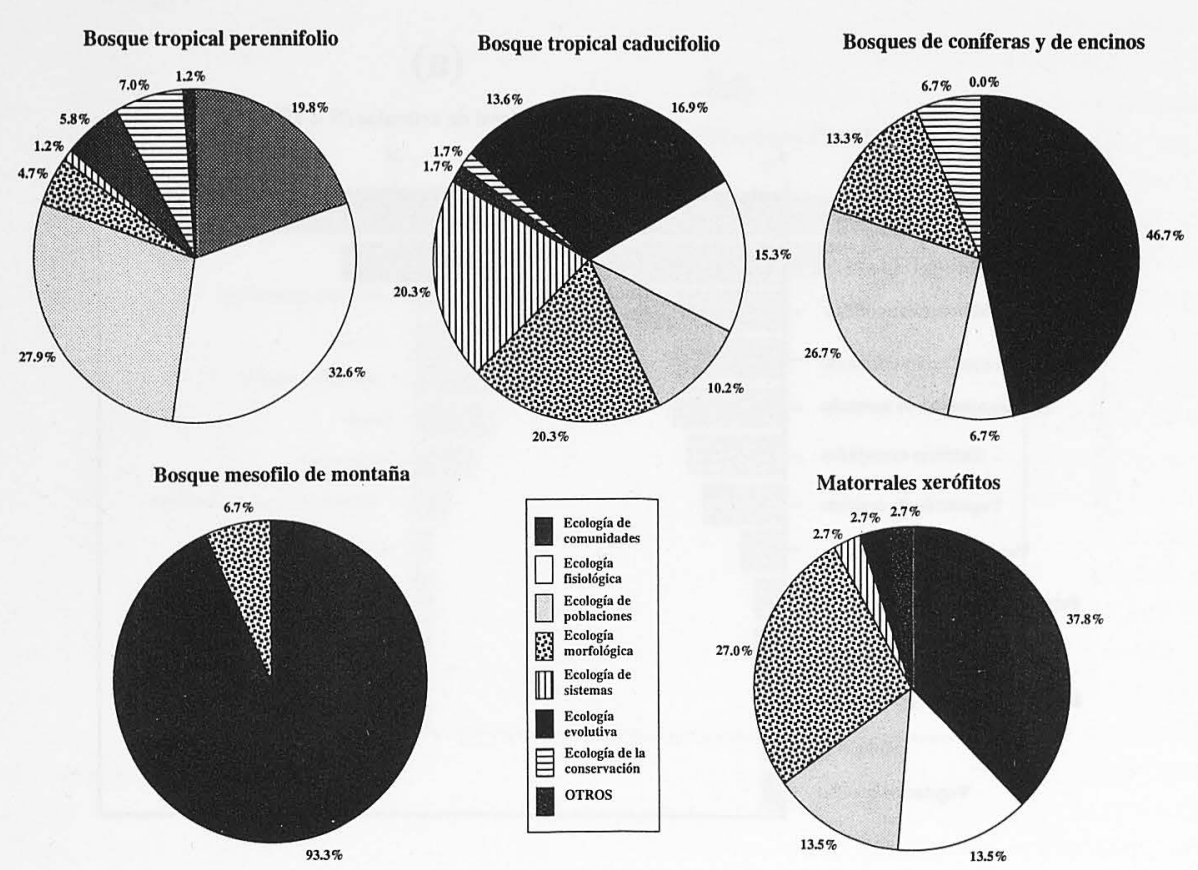

FIgURA 4. Representación porcentual de los artículos publicados abordando temas de diferentes áreas de la ecología de estudios desarrollados en tipos de vegetación más estudiados en México.

En la Figura 4 se muestra la proporción de artículos publicados, para cada uno de los cinco tipos de vegetación más estudiados, divididos por las áreas de estudios de ecología vegetal que ha continuación se detallan

\section{PRODUCCIÓN POR ÁREAS DE ESTUDIO}

La mayor actividad de investigación ha recaído principalmente en tres áreas de estudio (ecología fisiológica, ecología de poblaciones y ecología de comunidades) no obstante que en México se cultivan actualmente todas las áreas de la ecología vegetal (figura 3b). Un análisis histórico muestra que la productividad científica en las distintas áreas de estudio ha venido uniformizándose, de manera que la diversidad de temas de investigación ha aumentado de manera clara (Fig. 5). Así, mientras que la representación relativa del área de ecología de comunidades y ecología fisiológica han disminuido, otras áreas han aumentado en su representación, como es el caso de ecología de poblaciones, ecología evolutiva y ecología de la conservación y manejo de recursos. Otras son de muy reciente aparición, como es el caso de la ecología del paisaje. A continuación presento una descripción más detallada de la actividad lograda en las áreas de estudio más importantes.

\section{Ecología fisiológica}

Uno de los cuerpos de conocimiento más sólidos que se ha generado en México se refiere al estudio del significado ecológico de los mecanismos fisiológicos que regulan la germinación de semillas de plantas de bosque tropical perennifolio. Este campo, desarrollado por el grupo dirigido por Carlos Vázquez-Yanes y Alma Orozco-Segovia, ha aportado contribuciones originales sobresalientes. Como ejemplo de estos aportes queda el descubrimiento de que los mecanismos fotoblásticos y termoblástico que regulan la germinación de las semillas de algunas especies arbóreas de rápido crecimiento y que desempeñan para estas plantas un papel ecológico fundamental. Estos árboles sobreviven, crecen y se reproducen solo bajo condiciones de elevada disponibilidad de recursos lumínicos (Vázquez-Yanes, 1980) de manera que estos mecanismos fisiológicos permiten la germinación solo cuando existe una abundante entrada de energía solar hasta el nivel del suelo (Vázquez-Yanes y Orozco-Segovia, 1984).

Otro hallazgo de relevancia fue el descubrimiento de la existencia de plantas arbóreas con el metabolismo tipo 

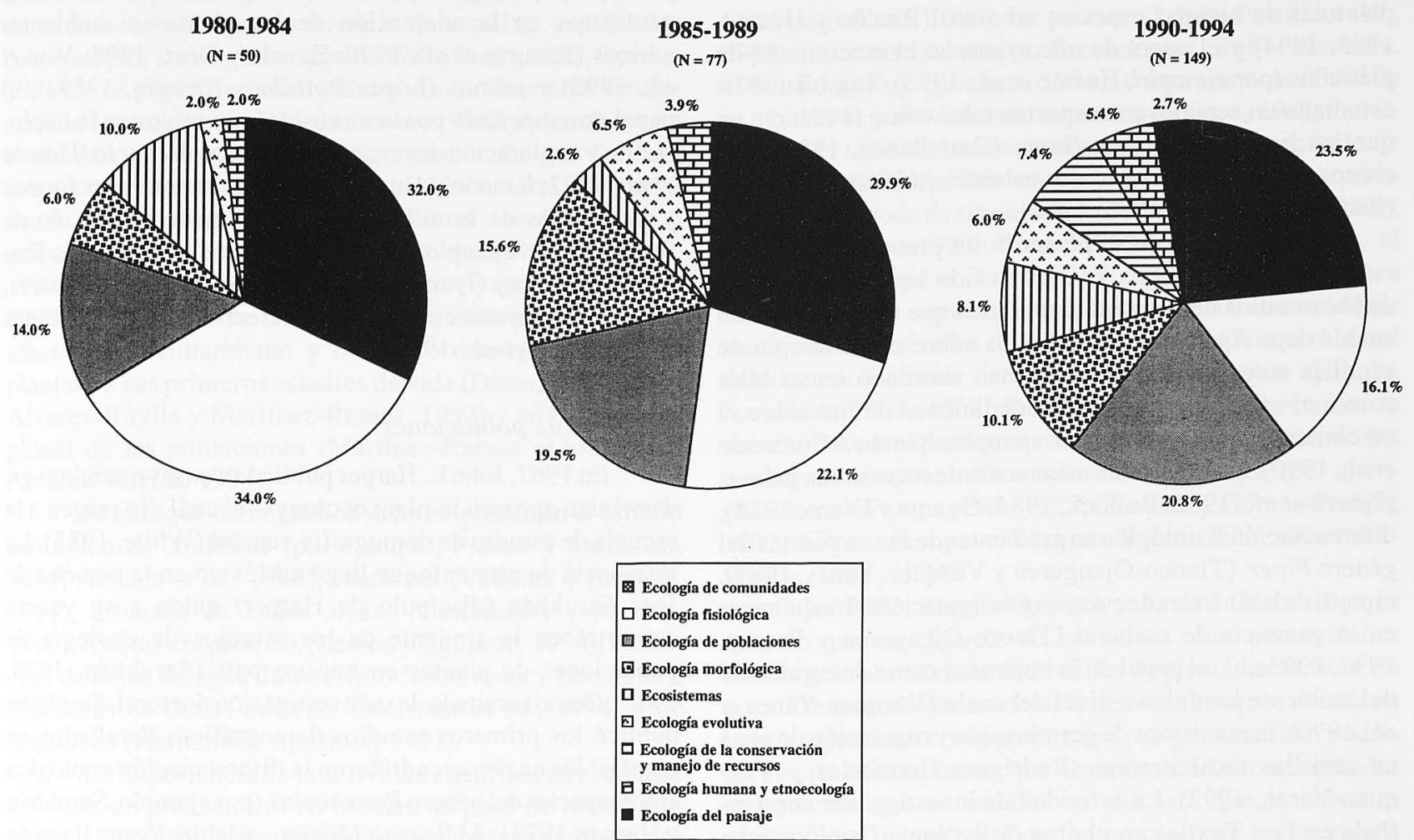

FIgURA 5. Cambio temporal en la proporción de artículos científicos publicados en diferentes áreas de estudio de la ecología vegetal en México.

CAM en bosques tropicales perennifolios. Este metabolismo permite fijar carbono en ausencia de luz (es decir, por la noche) y es característico de muchas especies de plantas suculentas y epífitas, típicas de ambientes xéricos. Este atributo fisiológico supera el problema del intercambio gaseoso durante el día, que surge porque estas plantas cierran sus estomas para reducir la perdida de agua al quedar expuestas a elevadas cargas de radiación solar. En 1983, Clara Tinoco-Ojanguren y Carlos Vázquez-Yanes registraron en hojas del árbol epífito del género Clussia, que es común en la selva de Los Tuxtlas, una reacción de acidificación y movimiento estomático típicas del metabolismo CAM. Esto implicaba descubrir que en ambientes supuestamente ilimitados en agua existen zonas (como los del dosel del bosque) en las cuales las plantas experimentan condiciones xéricas. Dos año después, este fenómeno fue redescubierto por colegas extranjeros en otra especie de Clussia encontrada en Brasil (Ting, et al., 1985). Aunque este autor no reconoció el hallazgo a Tinoco y Vázquez-Yanes, estudios recientes han puesto claramente de manifiesto la originalidad del trabajo realizado por los autores mexicanos (Vázquez-Yanes, 1995).

La importancia de las contribuciones de VázquezYanes y Orozco-Segovia queda también de manifiesto por la reciente contribución que hicieron al Annual Review of Ecology and Systematics (Vázquez-Yanes y Orozco-Segovia, 1993), que como sabemos es un espacio de publicación selecto, ofrecido a personas que han consolidado líneas de investigación.

El estudio de los mecanismos fisiológicos que permiten a las plantas ajustarse a las condiciones de su entorno inmediato es un campo que ha crecido en México durante los 
últimos cinco años. En el bosque tropical seco de Chamela, Jalisco, se ha estudiado el efecto de la restricción de nutrientes del suelo y de recursos lumínicos sobre el crecimiento de plántulas de algunas especies arbóreas (Rincón y Huante, 1993, 1994) y el papel de micorrizas en el crecimiento de plántulas (por ejemplo, Huante et al., 1993). También se ha estudiado en este bosque aspectos tales como la manera en que las lianas capturan carbono (Castellanos, 1991) y el comportamiento de estomas y balances hídricos en árboles (Fanjul y Barradas, 1985, 1987).

El bosque tropical perennifolio presente en la Estación de Biología de Los Tuxtlas ha sido sede de la mayoría de los estudios de ecología fisiológica que se han realizado en México. Aparte de los estudios sobre ecofisiología de semillas antes mencionados, se han abordado temas tales como: el efecto de diferentes condiciones de luz sobre el crecimiento de plántulas (por ejemplo, Sánchez-Coronado et al., 1990), estrategias de asignación de recursos en palmas (Piñero et al., 1982; Bullock, 1984; Oyama y Dirzo, 1988), diferenciación fisiológica en gradientes de luz en plantas del género Piper (Tinoco-Ojanguren y Vázquez-Yanes, 1983), el papel de la dinámica de estomas y de limitaciones bioquímicas en la ganancia de carbono (Tinoco-Ojanguren y Pearcy, 1992, 1993a,b), el papel de la hojarasca como determinante del ambiente lumínico a nivel del suelo (Vázquez-Yanes et al., 1990), mecanismos de germinación y regulación de agua en semillas recalcitrantes (Rodríguez-Hernández y Vázquez-Yanes, 1992). La actividad de investigación desarrollada en Los Tuxtlas en el área de ecología fisiológica ha estado acompañada por la organización de eventos académicos importantes a nivel internacional, tal como aquel que generó el libro Physiological ecology of plants in the wet tropics, considerado como importante en el área de estudio (Medina et al., 1984).

Algunos estudios llevados a cabo en otros sistemas vegetales son: crecimiento de plantas pioneras de dunas costeras bajo diferentes niveles de disponibilidad de nutrientes (Martínez y Rincón, 1993), estrategias de captación de agua de neblina en pinos (Barradas y Fanjul, 1983), efecto de la temperatura sobre la germinación en cactáceas (Cancino et al., 1993), dinámica de estomas y estrategia adaptativas en arbustos de zonas áridas (León-de la Cruz y Fanjul, 1985) y efecto de la luz y la temperatura en la germinación de semillas de plantas arvenses (Corkidi, et al., 1991).

\section{Ecología morfológica}

Estudios de índole morfológico, aunque aún escasos, han empezado a contribuir de manera importante al conocimiento de ecología vegetal en México. Así, se ha explorado el papel jugado por diferentes formas florales (por ejemplo, en plantas heteroestílicas) en el éxito reproductivo de las plantas (Pérez Nasser et al., 1993), el papel jugado por anexos florales (tales como los nectarios observados en el arbusto Croton suberosus y la orquídea Schomburgkia tibicinis) como órganos de atracción de insectos que parecen proteger a las plantas de posibles herbívoros (Domínguez et al., 1989) o bien que usan a las plantas como hábitat (Rico, 1989), el papel jugado por formas foliares, arquitecturales y geoformas en la adaptación de las plantas a ambientes xéricos (Ezcurra et al., 1990; Zavala y Díaz, 1995; Vite et al., 1995) y salinos (López-Portillo y Ezcurra, 1989a), el papel desempeñado por la morfología de raíces en la exploración y explotación de recursos minerales del suelo (Huante et al., 1992; Rincón y Huante, 1994), el papel de las formas y estructuras de semillas y diásporas en la dispersión de semillas (por ejemplo, Ibarra-Manríquez et al., 1991; Ibarra-Manríquez y Oyama, 1992) y el papel de formas foliares, tallos y metámeras en el habito de crecimiento de las lianas (Castellanos et al., 1989).

\section{Ecología de poblaciones}

En 1967, John L. Harper publicó su ensayo seminal «A darwinian aproach to plant ecology», el cuál dio origen a la escuela de estudio de demografía vegetal (White, 1985). La influencia de este enfoque llegó a México en la persona de José Sarukhán (discípulo de Harper) quién a su vez se convirtió en la simiente de los estudios de ecología de poblaciones de plantas en nuestro país (Sarukhán, 1978, 1980). Como resultado de su investigación doctoral, Sarukhán publicó los primeros estudios demográficos detallados en plantas, los cuales escudriñaron la diferenciación ecológica entre especies del género Ranunculus (por ejemplo, Sarukhán y Harper, 1973). Al llegar a México, estableció una línea de investigación que exploró mecanismos de regulación de poblaciones de especies arbóreas que habitan en sistemas boscosos contratantes (Sarukhán, 1978, 1980). En gran medida, esta línea de investigación fue impulsora de la considerable cantidad de trabajos que se han originado en México en el área de ecología de poblaciones (Fig. 3b). La proporción de artículos publicados en el área de ecología de poblaciones ha venido en aumento. La Figura 5 muestra que esta proporción ha crecido más del $50 \%$ durante los últimos 15 años.

Los demógrafos de plantas mexicanos han producido algunos de los estudios más completos del ciclo de vida de árboles tropicales conocidos en la literatura mundial. Estos estudios han explorado los factores que determinan las tasas de muertes y nacimientos en poblaciones de especies con historias de vida contrastantes (Sarukhán, 1980; Córdova, 1985; Calvo, 1989; Oyama, 1990; Alvarez-Buylla y Martínez-Ramos, 1992; Durán y Franco, 1993; Mendoza y Franco, 1993), y han analizado la dinámica de las poblaciones con modelos formales (Piñero, et al. 1984; Martínez-Ramos et al., 1988a; Palomeque, 1988; Calvo, 1989; AlvarezBuylla y García-Barrios, 1991; Durán, 1992; Alvarez-Buylla, 1994).

Otros estudios poblacionales han explorado el papel que juegan las interacciones bióticas como determinantes de los atributos demográficos de las plantas tropicales. En este contexto, se ha estudiado el papel de animales herbívoros en 
el rendimiento de las plantas (Dirzo, 1984; Núñez-Farfán y Dirzo, 1988, 1991; García-Franco et al., 1991; Oyama y Dirzo, 1991; Domínguez y Dirzo, 1992), de dispersores y depredadores en la sobrevivencia de semillas y plántulas (por ejemplo, Dirzo y Domínguez, 1986; Dirzo, 1987; Oyama, 1991) y el papel de polinizadores en la transición óvulosemilla (por ejemplo, Búrquez et al., 1988). Además, se ha explorado el efecto demográfico producido por factores asociados con eventos de perturbación natural, tal como aquel experimentado por las plantas al ser defoliadas o golpeadas por la caída de ramas y árboles (Mendoza et al., 1987; Martínez-Ramos et al., 1988b; Oyama y Mendoza, 1990). También se ha evaluado como estas perturbaciones afectan el reclutamiento y derrotero demográfico de las plantas en sus primeros estadios de vida (Dirzo, 1984, 1987; Alvarez-Buylla y Martínez-Ramos, 1992) y en la dinámica global de las poblaciones (Martínez-Ramos et al. 1988a; Alvarez-Buylla, 1994).

El enfoque demográfico se ha empleado en el estudio ecológico de coníferas (por ejemplo, Franco y Sarukhán, 1981; Arriaga et al., 1988), cactáceas (Valiente y Ezcurra, 1991), arbustos de zonas áridas (Castellanos y Molina, 1990), rosetas crasuláceas (Larson y Eguiarte, 1994), herbáceas de lagos de agua dulce (Niño-Sulkowska y Lot, 1983), herbáceas de dunas costeras (Martínez et al., 1994) y de orquídeas (Hernández-Apolinar, 1992).

La importancia de la actividad científica en el área de ecología de poblaciones en México se puso de manifiesto con el simposio internacional sobre ecología de poblaciones de plantas organizado por la Universidad Nacional Autónoma de México en 1982 en Oaxtepec, Morelos. Este evento se efectuó con motivo del centenario de la muerte de Charles Darwin y dio lugar al libro «Perspectives on plant population ecology» (Dirzo y Sarukhán, 1984), el cuál generó nuevas líneas de investigación y es de consulta obligada en cursos de posgrado a nivel mundial.

\section{Ecología de comunidades}

Una gran proporción de los estudios de ecología vegetal desarrollados en México inciden sobre temas de ecología de comunidades (Fig. 3b). Uno de los estudios clásicos del área es el de Miranda y Hernández-X (1963) quienes aportaron el primer sistema de clasificación de los tipo de vegetación en México. Este trabajo influyó de manera importante el desarrollo de la ecología vegetal en México, dando pie al análisis de la fisiognómia y atributos sinecológicos de diferentes tipos de vegetación.

Muchos de los estudios realizados en el área (Fig. 4) se han orientado al análisis cuantitativo de la estructura de las comunidades (por ejemplo, evaluando la composición florística, diversidad de especies, y organización de las plantas en la comunidad) y en la exploración de patrones espaciales de la vegetación. Se ha estudiado con este enfoque a comunidades de plantas de bosque de coníferas (e.g., Obieta y Sarukhán, 1981; de bosque mesófilo de montaña (e.g., Puig et al., 1983; Meave et al., 1992), de bosque tropical perennifolio (e.g., Piñero et al., 1977; Bongers et al., 1988) de bosque tropical caducifolio (e.g., Arriaga y León de la Cruz, 1989), de petenes (López-Portillo et al., 1989), de zonas áridas (e.g., Ezcurra et al., 1987; Montaña, 1990; Búrquez y Quintana, 1994), dunas costeras (Castillo et al., 1991) y de sistemas acuáticos (Quiroz, 1981)

Un tema de investigación que se ha desarrollado de manera notable en México es el estudio de la sucesión vegetal, sobre todo en el bosque tropical perennifolio (Sarukhán, 1981; Guevara, 1990; Dirzo, 1993). En un principio, el estudio se dirigió a describir los cambios temporales en composición y estructura que expresan las comunidades sucesionales derivadas de la eliminación del bosque natural debido a actividades de roza-tumba-quema (por ejemplo, Sarukhán, 1964, 1968; Purata, 1986). Posteriormente, se estudiaron estos procesos sucesionales como parte de los mecanismos regenerativos naturales del bosque (por ejemplo, Gómez-Pompa et al., 1972; Guevara y Gómez-Pompa, 1972; Gómez-Pompa y Vázquez-Yanes, 1985; MartínezRamos, 1985, 1994).

En 1972, Sergio Guevara y Arturo Gómez-Pompa notaron que en el suelo de las selvas altas perennifolias existía gran abundancia de semillas de plantas heliófilas. Se postuló entonces que en el suelo de estos bosques existía un banco regenerativo importante que, en gran medida, podría ser fuente de las plantas que componen a las comunidades secundarias que se establecen una vez que la selva es removida, y que es vital en los procesos de regeneración natural del bosque tropical perennifolio. Estos trabajos generaron una cascada de estudios sobre bancos de semillas en bosques tropicales perennifolios a nivel mundial (ver por ejemplo, Garwood, 1989). La naturaleza dinámica de estos bancos de semillas fue ignorada por casi dos décadas (PérezNasser y Vázquez-Yanes, 1986). Estudios experimentales realizados en México mostraron que este banco es muy dinámico y depende de manera importante de la renovación de las semillas del suelo a través de la producción de una abundante lluvia de semillas (Alvarez-Buylla y MartínezRamos, 1990; Alvarez-Buylla y García-Barrios, 1991).

La idea de que los bosques son entidades que se renuevan de manera cíclica, a través de procesos naturales de perturbación y regeneración, se ha establecido como un marco conceptual robusto, con el cual es posible explorar y entender aspectos ecológicos y evolutivos del bosque tropical perennifolio; los estudios realizados en México han ayudado a construir este marco (Martínez-Ramos, 1985, 1994; Martínez-Ramos y Soto-Castro, 1993; Arriaga, 1988a,b; Popma et al., 1988; Dirzo et al., 1992). En Los Tuxtlas se descubrió que ciertas palmas del sotobosque pueden usarse para establecer un eje temporal en el estudio de los procesos naturales de regeneración del bosque (Martínez-Ramos et al., 1988b). Tal herramienta permite explorar, a un tiempo dado, eventos poblacionales y comunitarios que ocurren a través de decenas y aún centenas de años (Sarukhán et al., 1985; Martínez-Ramos y Alvarez-Buylla, 1986; Alvarez- 
Buylla, 1994). Las perturbaciones humanas, que superan en escala y magnitud a aquellas que ocurren de manera natural en el bosque, conduce al desarrollo de los eventos sucesionales observados en áreas deforestadas y abandonadas, los cuales, en ocasiones, no culminan con una comunidad boscosa similar a la original (Purata et al., 1988). Esto último ocurre cuando se han deteriorado de manera severa los bancos regenerativos del bosque, por ejemplo, cuando la comunidad nativa es reemplazada por pastizales ganaderos. En este contexto, se están desarrollando estudios que tratan de detectar factores que pueden promover, en tales circunstancias de deterioro, la recuperación del bosque (e.g., Guevara et al., 1986; Guevara y Laborde, 1993).

El estudio de la dinámica de comunidades de plantas de zonas áridas empieza a establecerse como un tema de investigación importante en México, sobre todo debido a los trabajos que han abordado el estudio del papel del nodricismo y la competencia en la estructuración de la comunidad y en la dinámica de reemplazamiento temporal entre especies (Valiente et al., 1991a,b; Arriaga et al., 1993; Flores-Martínez et al., 1994). Otros aspectos estudiados a nivel de la comunidad se refieren a procesos fenológicos de las comunidades (Carabias y Guevara, 1985, López-Portillo y Ezcurra, 1985; Bullock y Solís-Magallanes, 1990; Ibarra-Manríquez et al., 1991, Ibarra-Manríquez y Oyama, 1992; Maya y Arriaga, 1995), patrones de niveles de daño foliar por herbívoros (Dirzo et al., 1987; De la Cruz y Dirzo, 1988; Filip et al., 1995), el papel jugado por herbívoros en la sucesión vegetal (Núñez-Farfán y Dirzo, 1985), procesos de sucesión secundaria en bosques de pino-encino (González-Espinosa et al., 1991), zonación de la vegetación debido a la interacción entre especies (López-Portillo y Ezcurra, 1989b; Montaña et al., 1990) y aspectos de dispersión y depredación de semillas en comunidades de nopaleras (González-Espinosa y Quintana-Ascencio, 1986).

\section{Ecosistemas}

Los estudios a nivel de ecosistemas han surgido primordialmente del proyecto de largo plazo sobre ecología de cuencas desarrollado en la Estación de Biología Experimental «Chamela» (Jalisco), iniciado en el año de 1981 (Sarukhán y Maass, 1990). Este proyecto ha dado lugar a descripciones detalladas sobre patrones de producción, caída y descomposición de hojarasca (Martínez-Yrizar y Sarukhán, 1990, 1993 ), de cambios temporales en el área fotosintética del bosque (Maass et al., 1995), estimaciones de la biomasa vegetal sobre y bajo el suelo (Castellanos et al., 1991; Martínez-Yrizar et al., 1992), de la productividad primaria neta (Martínez-Yrizar et al., 1995) y análisis de flujos biogeoquímicos (García-Méndez et al., 1991; Jaramillo y Sandford, 1995). El proyecto cuencas pretende caracterizar flujos energéticos y biogeoquímicos que ocurren en el bosque de Chamela para posteriormente evaluar el efecto que tendrían perturbaciones producidas por diferentes actividades agropecuarias sobre el ecosistema (Sarukhán y Maass,
1990). En este sentido, se ha estudiado el efecto que tiene la transformación del bosque en potreros sobre la erosión, pérdida de nutrientes y dinámica de carbono del suelo (García-Oliva et al., 1994, 1995a,b; Maass et al., 1988).

En el bosque perennifolio de Los Tuxtlas, se han desarrollado estudios sobre productividad primaria, en términos de producción de hojarasca y su descomposición en el suelo (Alvarez-Sánchez y Guevara, 1985, 1993; AlvarezSánchez, 1991). En zonas áridas aún no se han desarrollado estudios a nivel del ecosistema pero existen algunos que proveen información importante a esta área de la ecología (e.g., Ezcurra y Rodríguez, 1986; Montaña et al., 1988; Maya y Arriaga, 1995)

\section{Ecología evolutiva}

Esta es un área de muy reciente aparición en México, la cuál se ha derivado de grupos de investigación que originalmente cultivaron el área de ecología de poblaciones. Daniel Piñero desarrolló la primera línea de investigación en el área con sus estudios de ecología evolutiva del género Phaseolus (e.g., Piñero y Eguiarte, 1987) y sus bacterias simbiontes del género Rhizobium (Valdés y Piñero, 1992). Los estudios demográficos en plantas tropicales han dado pie al estudio de fuentes de variabilidad intrapoblaciónal en adecuación, genética de poblaciones y microevolución en sistemas tropicales (Piñero y Sarukhán, 1982; Piñero et al., 1982; Sarukhán et al., 1984; Oyama y Dirzo, 1988, 1990; PérezNasser et al., 1993; Eguiarte et al. 1993; Alvarez-Buylla y Garay, 1995). Larry Venable, actualmente en la Universidad de Arizona, impulsó en México el campo de estudio de ecología evolutiva y genética cuantitativa con sus estudios en la planta ruderal Heterosperma pinnatum (Venable , 1984).

Otras líneas de investigación han abordando el estudio de las presiones de selección ejercidas por herbívoros sobre las plantas (Núñez-Farfán y Dirzo, 1994a) y aquellas ejercidas por la predecibilidad lluvia en la fenología de la floración (Domínguez y Dirzo, 1995) y tiempos de emergencia de plántulas (Núñez-Farfán et al., 1994b). También se ha estudiado el papel de sistemas reproductivos y tamaños poblacionales en la estructura genética de las poblaciones (por ejemplo, Eguiarte et al., 1992, 1994; Pérez-Nasser et al., 1993; Escalante et al., 1993).

\section{Otras áreas}

Además de los estudios de las líneas básicas de la ecología ya detalladas, otras áreas están empezando a cobrar importancia. Así el fuerte impulso dado por Victor Manuel Toledo a los estudios sobre etnoecología ha dado lugar al surgimiento en México de la revista Etnoecológica (Toledo 1992). El área de la llamada ecología química, tratados en extensión por Anaya en el presente número, también es un área en actividad de la ecología vegetal en México. El estudio de los patrones y procesos relacionados con la distribución de especies y comunidades a nivel de paisajes 
y el aporte de estos estudios en la toma de decisiones en la conservación y manejo de la vegetación, es un área de estudio que se encuentra en sus inicios (Jardel, 1990; Dirzo y Miranda, 1991; Dirzo, 1991, 1993; González-Espinosa et al., 1995; Bojórquez-Tapia et al., 1994). También están ya surgiendo estudios que señalan la aplicación de los estudios de ecología básica en el manejo sustentable y conservación de los recursos (por ejemplo, Jardel y Sánchez-v'elázquez, 1989; Del Amo, 1991; Alvarez-Buylla, 1994; Alvarez-Buylla y García-Barrios, 1991; Eguiarte et al., 1993; Oyama, 1993; Olmsted y Alvarez-Buylla, 1995).

\section{PERSPECTIVAS}

La revisión aquí presentada muestra que el campo de ecología vegetal en México se encuentra en un proceso activo de desarrollo, cubriéndose una gran variedad de líneas de investigación en las diferentes áreas de estudio de la disciplina. Algunas de estas líneas se han consolidado, generando conocimientos de relevancia universal. Entre estas pueden mencionarse los estudios de ecofisiología de semillas y los estudios de ecología de poblaciones y comunidades de plantas tropicales. Los estudios de ecosistemas en cuencas y los de ecología de poblaciones y comunidades de plantas en zonas áridas están cobrando ya este estatus. En este sentido puede decirse que existe ya una escuela mexicana de ecología vegetal. A mi parecer, el desarrollo y maduración de esta escuela depende, de manera importante, en que las líneas consolidadas se sigan cultivando y apoyando, aprovechando la gran infraestructura de conocimientos ya obtenida. Igualmente importante es la generación recursos humanos que renueven y mejoren la calidad académica de los actuales grupos de investigación. La existencia en México de varios posgrados en ecología, con elevados estándares académicos, puede asegurar que este proceso de renovación se lleve a cabo (ver Moreno-Casasola y Sánchez, 1990 y Moreno-Casasola, este número).

En gran medida, las líneas consolidadas se han desarrollado bajo el apoyo de las estaciones biológicas de campo (Guevara, 1990). Considero que las estaciones biológicas deben seguir desempeñando un papel preponderante en el desarrollo de los estudios de ecología vegetal. Algunas de las bondades que ofrecen las estaciones son: i) el resguardo de los sistemas y equipo de estudio, lo que minimiza el riesgo de perturbaciones no deseadas al material bajo investigación, ii) el desarrollo de nuevos proyectos con antecedentes sólidos, dado que en las estaciones se genera un proceso importante de agregación de conocimientos, iii) la generación de una dinámica importante de intercambio de ideas entre grupos de investigación con diversos temas de estudio, iv) la posibilidad de llevar a cabo experimentos naturales controlados, v) la posibilidad de ganar niveles de conocimientos más profundos a través de proyectos multidisciplinarios, y vi) la posibilidad de llevar cabo proyectos de investigación y difusión que incidan sobre la conservación y manejo de recursos naturales en cooperación con las comunidades humanas que dependen o interactuan con tales recursos.

En México, se han establecido estaciones biológicas en casi todos los tipos de vegetación presentes en el país (Cuadro 2). Estas estaciones poseen la infraestructura suficiente para apoyar estudios en todas las áreas de estudio de la ecología. Hasta ahora, sin embargo, no se han explotado a plenitud las bondades ofrecidas por las estaciones biológicas. La mayoría de los estudios realizados en las estaciones se han desarrollado de manera más o menos independiente, dejando de lado la posibilidad de maximizar el nivel de entendimiento de los fenómenos, ahorrando esfuerzos y costos. Un procedimiento que puede ayudar a resolver este problema es el establecimiento de «planes maestros» de las estaciones biológicas, que orienten y coordinen el desarrollo de los proyectos de investigación hacia partes del conocimiento que no se tienen a que se encuentran aún pobremente entendidas. En este sentido, creo que un componente primordial para el desarrollo futuro de la ecología vegetal en México es el establecimiento y operación de estos planes.

Los fenómenos ecológicos, principalmente aquellos que ocurren por arriba del nivel de organismos individuales, generalmente se desenvuelven en escalas amplias de espacio y de tiempo. Por esto, los estudios ecológicos de largo plazo y de gran escala son primordiales para entender dichos fenómenos. Los planes maestros de las estaciones (y los de nuestras instituciones en general) deberían impulsar el establecimiento de proyectos de esta índole. Partes fundamentales de los mismos son: inventarios detallados de flora y fauna, inventarios detallados del ambiente abiótico (tales como registros climáticos y micrometereológicos detallados y caracterización fina de la topográfica y suelos), establecimiento de parcelas fijas en las cuales se de el seguimiento cuidadoso de la dinámica de poblaciones y comunidades de plantas y animales, así como del funcionamiento del ecosistema. Estas parcelas podrían ser los sitios comunes de estudio de proyectos con diversos enfoques e inquietudes de investigación. Bien diseñada y estructurada, la actividad científica concentrada en estas parcelas puede ayudar a entender y resolver problemas ecológicos (y biológicos en general) básicos y de conservación y manejo de recursos naturales.

Un nivel de organización aún mayor es la posibilidad de crear una red de proyectos ecológicos de largo plazo en estaciones de campo que resguardan sistemas ecológicos contrastantes. Planes maestros de investigación de estas estaciones que sean compatibles en objetivos, sistemas de estudio y métodos de investigación pueden agilizar nuestra capacidad para comprender fenómenos ecológicos, que trascienden una escala meramente local. El éxito de todas estas empresas depende de nuestra capacidad para obtener nuevos recursos y, de manera muy importante, de nuestra habilidad para detectar y establecer proyectos de investigación con alto valor intelectual, cuidadosamente diseñados para responder a preguntas relevantes, de amplio interés académico y social. 
CuAdro 2. Algunas de los lugares donde existen estaciones biológicas de campo en México, en las que es posible llevar a cabo estudios ecológicos de largo plazo.

\begin{tabular}{|c|c|c|}
\hline Estación biológica & Tipo de vegetación & Localización \\
\hline $\begin{array}{l}\text { «Los Tuxtlas» } \\
\text { (UNAM) }\end{array}$ & Bosque tropical perennifolio & Veracruz. \\
\hline $\begin{array}{l}\text { «Chajul» } \\
\text { (UNAM-Conservación Internacional) }\end{array}$ & Bosque tropical perennifolio & Chiapas. \\
\hline $\begin{array}{l}\text { «Chamela» } \\
\text { (UNAM) }\end{array}$ & Bosque tropical caducifolio & Jalisco. \\
\hline $\begin{array}{l}\text { «El Pedregal de San Angel» } \\
\text { (UNAM) }\end{array}$ & Matorral xerófilo & México, D.F. \\
\hline $\begin{array}{l}\text { «Rancho del Cielo» } \\
\text { (Universidad de Tamaulipas) }\end{array}$ & Bosque mesófilo de montaña & Tamaulipas \\
\hline $\begin{array}{l}\text { «La Michilia» } \\
\text { (Instituto de Ecología, A.C.) }\end{array}$ & Bosques de coniferas y encinares & Durango \\
\hline $\begin{array}{l}\text { «Mapimi» } \\
\text { (Instituto de Ecología, A.C.) }\end{array}$ & Matorral xerófilo & Durango \\
\hline $\begin{array}{l}\text { «La Mancha» } \\
\text { (Instituto de Ecología) }\end{array}$ & Vegetación costera y de dunas & Veracruz \\
\hline $\begin{array}{l}\text { «El Triunfo» } \\
\text { (Instituto de Historia Natural, Chis.) }\end{array}$ & Bosque mesófilo de montaña & Chiapas \\
\hline $\begin{array}{l}\text { «Las Joyas» } \\
\text { (Instituto Manantlán, } \\
\text { Universidad de Guadalajara) }\end{array}$ & Bosque de coníferas y mesófilo de montaña & Jalisco \\
\hline «Zapotitlán» & Matorral xerófilo & Puebla \\
\hline $\begin{array}{l}\text { «Zoquiapan» } \\
\text { (Instituto Nacional de } \\
\text { nvestigaciones Forestales) }\end{array}$ & Bosque de coníferas & Edo. de México. \\
\hline
\end{tabular}

Como muchas disciplinas científicas, la ecología vegetal procede a través de la descripción de patrones y el entendimiento de los procesos y mecanismos que originan tales patrones. La ecología vegetal en México se ha concentrado fundamentalmente en la descripción de patrones y, en menor grado, del estudio de los procesos. Esto es evidente si notamos que la gran mayoría de los estudios mostrados en la figura 1 fueron dirigidos a caracterizar estructuras (de plantas, poblaciones o comunidades), a través de enfoques empíricos. En el futuro se debe impulsar la búsqueda de los principios (mecanismos) que dan lugar a los procesos. En este sentido, es fundamental pasar de la etapa descriptiva al uso de la experimentación como medio para detectar tales principios. La obtención de este conocimiento no sólo es parte del objetivo de la actividad científica sino que es la base para generar tecnología útil a la sociedad. Creo que la capacidad para lograr tal conocimiento puede también de- pender de fortalecer nuestra escuela con el enfoque deductivo. La teoría, que genera hipótesis que pueden refutarse a través de la experimentación, es una herramienta poderosa en el desarrollo científico. Si de algo necesitamos los ecólogos en México, son de colegas formados con una mentalidad deductiva. Basta mencionar que de los 277 artículos que usé en mi análisis, sólo cinco $(1.8 \%)$ pueden ubicarse dentro de lo que llamaríamos «ecología teórica» (e.g., Alvarez-Buylla, 1994).

Lo anterior no quiere decir que dejemos de describir y de usar el enfoque empírico. Este es importante pero, a mi parecer, la descripción es una posible etapa inicial del camino que finalmente se dirige, en ecología, hacia la búsqueda de las causas (principios) que determinan la abundancia y distribución de los seres vivos. Quiero finalizar este ensayo haciendo una rearreglo al texto que escribió J.L. Harper en el prefacio al libro Perspectives on plant population ecology (Dirzo y Sarukhán, 1984): «en México se encuentra 
un banco de plantas, que se exhibe con toda su variación y profusión, y también se encuentra un grupo vigoroso de ecólogos -especialmente botánicos- que se encuentra trabajando en este extraordinario banco».

\section{AGRADECIMIENTOS}

Quisiera agradecer sobremanera a L. Arriaga, C. Montaña, L. Sánchez, E. Rincón, C. Vázquez-Yanes, R. Dirzo, C. Domínguez y J. Nuñez-Farfán, su amable ayuda en la obtención de literatura. A la Sociedad Botánica de México por la invitación a escribir el presente ensayo y a K. Oyama su paciente y fina labor editorial.

\section{LITERATURA CITADA}

Alvarez-Sánchez J. 1991. Productividad primaria neta en una selva tropical húmeda. Bol. Soc. Bot. México 51: 3-12.

Alvarez-Sánchez J. Guevara S. 1985. Caída de hojarasca en la selva. En: Gómez-Pompa A, del Amo R S, edrs. Investigaciones sobre la regeneración de selvas altas en Veracruz, México. Alhambra, México, 171-190.

Alvarez-Sánchez J, Guevara S. 1993.Litterfall dynamics in a Mexican lowland tropical rain forest. Trop. Ecol. 34: 127-142.

Alvarez-Buylla E. 1994. Density dependence and patch dynamics in tropical rain forests: matrix models and applications to a tree species. Am. Nat. 143: 155-191.

Alvarez-Buylla E, Garay A. 1995. Population genetic structure of Cecropia obtusifolia, a tropical pioneer tree species. Evolution 48: 437-453.

Alvarez-Buylla E, García-Barrios R. 1991. Seed and forest dynamics: a theoretical framework and an example from the neotropics. Am. Nat. 137: 133-154.

Alvarez-Buylla E, Martínez-Ramos M. 1990. Seed bank versus seed rain in the regeneration of a tropical pioneer tree. Oecologia 84: 314-325.

Alvarez-Buylla E, Martínez-Ramos M. 1992. The demography of a neotropical pioneer tree: an evaluation of the pioneer-climax paradigm. J. Ecol. 80: 275-290.

Arriaga L. 1988a. Gap dynamics of a tropical cloud forest in Northeastern México. Biotropica 20: 178-184

Arriaga L. 1988a. Natural disturbance and treefalls in a pine oak forest on the Peninsula of Baja California, México. Vegetatio 78: 7379.

Arriaga I, Franco M, Sarukhán. 1988. Identification of natural groups of trees in uneven-aged forest using multivarate methods. J. Ecol. 76: 1092-1110.

Arriaga L, León de la Cruz JL. 1989. The tropical deciduous forest of Baja California Sur, México: a floristic and vegetational analysis. Vegetatio 84: 45-52.

Arriaga L, Maya M, Díaz S, Cancino J. 1993. Association between cacti and nurse perennials in a heterogeneous tropical dry forest in northwestern Mexíco. J. Veg. Sci. 4: 349-356.

Barradas V, Fanjul L. 1983. Capacidad de captación de agua a partir de la niebla en Pinus montezumae Lambet de la región de las Grandes Montañas del estadode Veracruz, México. Biótica 8: 303-307.

Bojórquez-Tapia L, Balvanera P, Cuarón A.1994. Biological inventories and computer database: their role in environmental assessments. Environ. Manag. 18: 775-785.

Bongers F, Popma J, Meave J, Carabias J. 1988. Structure and composition of the lowland rain forest of Los Tuxtlas, Mexico. Vegetatio 74: 55-88.

Bullock SH. 1984. Biomass and nutrient allocation in a neotropical dioecious palm. Oecologia 63: 426-428.

Bullock SH, Solís-Magallanes. 1990. Phenology of canopy trees of a tropical deciduous forest in Mexico. Biotropica 22: 22-35.

Búrquez A, Sarukhán J, Pedroza A. 1988. Floral biology of a primary forest palm, Astrocaryum mexicanum Liebm. Bot. J. Linn. Soc. 94: 407-419.

Búrquez A, Quintana MA. 1994. Islands of diversity: ironwood ecology and the richness of perennials in a Sonoran Desert biological reserve. En: An ecological and cultural keystone of the Sonaran Desert. Occasional Papers in Conservation Biology. Occasional paper No. 1. Conservation International, Washington, 9-27.

Calvo LM.1989. Demografía y dinámica de poblaciones de Aphelandra aurantiaca (Acanthaceae) en Los Tuxtlas, Veracruz. Tesis de licenciatura. México: UNAM.

Cancino J, León de la Cruz JL, Coria R, Romero H. 1993. Effect of heat treatment on germination of seeds of «cardon» (Pachycereus pringlei S. Wats. Britt. \& Rose, Cactaceae).J. Arizona-Nevada Acad. Sci. 27: 49-54.

Carabias J, Guevara S. 1985. Fenología de una selva tropical húmeda y en una comunidad derivada; Los Tuxtlas, Veracruz. En: Gómez-Pompa A, Del amo S, edrs. Vol II. Investigaciones sobre la regeneración de selvas altas en Veracruz, México Alhambra, México, 27-66

Castellanos A. 1991. Photosynthesis and gas exchange of vines. En: Putz FE, Mooney HA, edrs. The biology of vines. Cambridge University Press, 181-204.

Castellanos A, Molina F. 1990. Differential survivorship and establishment in Simmondsia chinensis (jojoba). J. Arid Environ. 19: $65-76$.

Castellanos J, Maass JM, Kummerow J. 1991. Root biomass of a dry deciduous tropical forest in Mexico. Plant Soil 131: 225-228.

Castellanos J, Mooney HA, Bullock SH, Jones C, Robichaux R. 1989. Leaf, stem and metamer characteristics of vines in a tropical deciduos forest in Jalisco, Mexico. Biotropica 21: 4149.

Castillo S, Popma J, Moreno-Casasola P. 1991. Costal sand dune vegetation of Tabasco and Campeche, Mexico. J. Veg. Sci. 2: 73-88.

Córdova B. 1985. Demografía de árboles tropicales. En: GómezPompa A, del Amo R S, edrs. Vol II. Investigaciones sobre la regeneración de selvas altas en Veracruz, México. Alhambra, México, 103-128.

Corkidi L, Rincón E, Vázquez-Yanes C. 1991. Effects of light and temperature on germination of heteromorphic achenes of Bidens odorata (Asteraceae). Can. J. Bot. 71: 1952-1958.

De la Cruz M, Dirzo R. 1988. A survey of the standing levels of herbivory in seedlings from a Mexican rain forest. Biotropica 19: 98-106.

Del Amo S. 1991. Management of secondary vegetation for artificial creation of useful rain forest in Uxpanapa, Veracruz, Mexico -an intermediate alternative between transformation and modification. En: Gómez-Pompa A, Whitmore TC, Hadley H, edrs. Rain forest regeneration and management. MAB, The Parthenon Publising group, París, 343-350.

Dirzo R. 1984. Herbivory: a phytocentric overview. En: Dirzo R, Sarukhán J, edrs. Perspectives on plant population ecology. Sinauer Sunderland, Massachusetts, 141-165.

Dirzo R. 1987. Estudios sobre interacciones planta-herbívoro en «Los Tuxtlas», Veracruz. Rev. Biol. Trop. (supl. 1) 35: 119-132. 
Dirzo R. 1991. Diversidad florística y estado de conservación de las selvas tropicales en México. En: Sarukhán J, Dirzo R, compiladores México ante los retos de la biodiversidad. Comisión Nacional para el Conocimiento y Uso de la Biodiversidad, México, 283-290.

Dirzo R. 1993. La ecología vegetal en México: resumen histórico , logros y perspectivas. En: Guevara S, Moreno- Casasola P, Rzedowski J, edrs Logros y perspectivas del conocimiento de los recursos vegetales de México en visperas del siglo XX1. Instituto de Ecología, A.C. y Sociedad Botánica de México, México, 125-134

Dirzo R, Sarukhán J, edrs. 1984. Perspectives on plant population ecology. Sinauer, Massachusetts.

Dirzo R, Domínguez. 1986. Seed shadows, seed predation and the advantages of seed dispersal. En: Estrada A, Fleming TH, edrs. Frugivores and seed dispersal. Dr. Junk Publishers, Dordrecht, 237-249

Dirzo R, Miranda A. 1991. Altered patterns of herbivory and diversity in the forest understory: a case of the possible causes of contemporary defaunation. En: Price PW, Lewinsohn TM, Fernández GW, Benson WW. Plant-animal interactions: evolutionary ecology in tropical and temperate forests. New York, Wiley, 273-297.

Dirzo R, Horvitz C, Quevedo H, López MA. 1992. The effects of gap size and age on the understory herb community of a tropical Mexican rain forest. J. Ecol. 80: 809-822.

Domínguez C, Dirzo R. 1992. Effects of defoliation on Erythroxylon havanense, a tropical proleptic species. Ecology 75: 18961902.

Domínguez C, Dirzo R. 1995. Rainfall and flowering synchrony in a tropical shrub: variable selection on the flowering time of Erytroxylon havanense. Evol. Ecol. 9: 201-216.

Domínguez C, Dirzo R, Bullock SH. 1989. On the function of floral nectar in Croton suberosus (Euphorbiaceae). Oikos 56: 109114.

Durán R. 1992. Variabilidad intra específica y dinámica poblacional de Pseudophoenix sargentii. Tesis doctoral. Centro de Ecología, UNAM. Mèxico.

Durán R, Franco M. 1992. Estudio demográfico de Pseudophoenix sargentii. Bull. l'Inst. Francais d'est. Andines 21: 609-621.

Eguiarte L, Parra V, Vargas F. 1994. Biología reproductiva y tamaño efectivo en Echeveria gibbiflora; un homenaje a Sewall Wright. En: Rojo A. Compilador Reserva ecológica «El Pedregal» de San Angel: ecología, historia natural y manejo. UNAM, México, 87-204.

Eguiarte L, Pérez-Nasser N, Piñero D. 1992. Genetic structure, outcrossing rate and heterosis in Astrocaryum mexicanum (tropical palm): implications for evolution and conservation. Heredity 69: 217-228.

Eguiarte L, Bórquez A, Rodríguez J, Martinez-Ramos M, Sarukhán J,Piñero D. 1993. Direct and indirect estimates of neighborhood and effective population size in a tropical palm Astrocaryum mexicanum. Evolution 47: 75-87.

Escalante A, Coello G, Soberón J. 1993.Lack of genetic variability in Lacandonia schismatica in its only known locality. Ann. Missouri Bot. Gard. 80: 898-901.

Ezcurra E, Rodríguez. 1986. Rain fall patterns in the Gran Desierto, Sonora, Mexico. J. Arid Environ. 10: 13-28.

Ezcurra E, Equihua M, López-Portillo J. 1987. The desert vegetation of the Pinacate, Sonora, Mexico. Vegetatio 71: 49-61.

Ezcurra E, Montaña C, Arriaga S. 1990. Architecture, light interception and distribution of Larrea species in the Monte Desert, Argentina. Ecology 72: 23-34.

Fanjul L, Barradas V. 1985. Stomatal behaviour of two heliophile understorey species of a tropical deciduous forest in Mexico. J. Appl. Ecol. 22: 943-954.

Fanjul L, Barradas V. 1987. Diurnal and seasonal variations in the water relations of some deciduous and everegreen trees of a deciduous forest. J. Appl. Ecol. 24: 289-303.

Filip V, Dirzo R, Maass JM, Sarukhán J. 1995. Levels of damage on the foliage of trees from a tropical deciduous forest in west Mexico. Biotropica (en prensa).

Franco M, Sarukhán J. 1981. Un modelo de la productividad forestal de un bosque de pino. Serie Premio Nacional Forestal (S.A.R.H.) 1: 1-71.

Flores O, Gerez P. 1988. Conservación en México: síntesis sobre vertebrados terrestres, vegetación y uso del suelo. INIREB, Conservación Internacional, México.

Flores-Martínez A, Ezcurra E, Sánchez Colón S. 1994. Effect of Neobuxbaumia tetetzo on growth and fecundity of its nurse plant Mimosa luisiana. J. Ecol. 82: 325-330.

García-Franco JG, Rico V, Zayas O.1991. Seed and seedling predation of Bromelia pinguin L. by the red land crab Gecarcinus lateralis Frem. in Veracruz, Mexico. Biotropica 23: 96-97.

García-Méndez, Maass JM, Marson PA, Vitousek PM. 1991. Nitrogen transformations and nitrous oxide flux in a drought-deciduous forest in Chamela, Jalisco, Mexico. Oecologia 88: 362-366.

García-Oliva F, Casar I, Morales P, Maass JM. 1994. Forest-topasture conversion influences on soil organic carbon dynamics in a tropical deciduous forest. Oecologia 99: 392-396.

García-Oliva F, Martínez R, Maass JM. 1995a. Long-term soil erosion as determined by Cs- 137 redistribution in a natural and perturbed tropical deciduous forest ecosystem. Geoderma (en prensa).

García-Oliva F, Martínez R, Maass JM. 1995b. Soil ${ }^{137}$ Cs activity in a tropical deciduous ecosystem under pature convertion in Mexico.

Garwood NC. 1989. Tropical soil seed banks: a review. En: Leck LA, Simson RL, Parker VT, edrs. Ecology of seed banks. USA: Academic Press, 149-190.

Gómez-Pompa A, Del Amo R S, edrs.1985. Investigaciones sobre la regeneración de selvas altas en Veracruz, México, Vol. II. Alhambra, México.

Gómez-Pompa A, Vázquez-Yanes C. 1985. Estudios sobre la regeneración de selvas en regiones cálido-húmedas de México. En: Góomez-Pompa A, del Amo R S, edrs. Vol II Investigaciones sobre la regeneración de selvas altas en Veracruz, México. Alhambra, México, 191-239.

Gómez-Pompa A, Vázquez-Yanes C, Guevara S. 1972. The tropical rain forest a nonrenewable resource. Science 177: 762-765.

Gómez-Pompa A, Vázquez-Yanes C, Del Amo S, Butanda A, edrs. 1976. Investigaciones sobre la regeneración de selvas altas en Veracruz, México. México: CECSA, CNEB, INIREB.

González-Espinosa M, Quintana-Ascencio PF.1986. Seed predation and dispersal in a dominant desert plant: Opuntia, ants, birds, and mammals. En: Estrada A, Fleming TH, edrs. Frugivores and seed dispersal. Dordrecht: Dr. Junk Publishers, 273-284.

González-Espinosa M, Quintana-Ascencio PF, Ramírez-Marcial N, Gaytán-Gúzman. 1991. Secondary succession in disturbed Pinus-Quercus forests in the highlands of Chiapas, Mexico.J. Veg. Sci. 2: 351-360.

González-Espinosa M, Ochoa-Gaona S, Ramírez-Marcial N, Quintana-AscencioPF.1995. Current land-use trends and conservation of old-growth forest habitats in the highlands of Chiapas, Mexico.En: Wilson M, Sander S, Estrada A, edrs. Conservation of neotropical migrant birds in Mexico. Washington: Smithsonian Institution Press, (en prensa). 
Guevara S. 1990. Historia de la ecología terrestre en México. Ciencias (No. Especial) 4: 89-93.

Guevara S, Gómez-Pompa A. 1972. Seeds from surface soils in a tropical region of Veracruz, Mexico. J. Arnold Arbor. 53: 312335.

Guevara S, Purata, Van Der Marel E. 1986. The role of remnant trees in tropical secondary succession. Vegetatio 66: 74-84.

Guevara S, Laborde J. 1993. Monitoring seed dispersal at isolated standing trees in tropical pastures: consequences for local species availability. Vegetatio 108: 319-338.

Harper JL. 1967. A Darwinian approach to plant ecology. J. Ecol. 55: 247-270.

Hernández-Apolinar M.1992. Dinámica poblacional de Laelia speciosa (HBK) Schltr. (Orchidaceae). Tesis de Licenciatura. Facultad de Ciencias, UNAM.

Huante P, Rincón E, Gavito M. 1992. Root system analysis of seven tree-seedling species from a tropical dry forest. Trees-Struct. Func. 6: 77-82.

Huante P, Rincón E, Allen E. 1993. Effect of vesicular arbuscular mycorrhizae on seedling growth of four tree species from the tropical deciduous forest in Mexico. Mycorrhiza 2: 141-145.

Ibarra-Manríquez G, Sánchez-Garfias B, González- García L. 1991. Fenología de lianas y árboles anemócoros en una selva cálido-húmeda de México. Biotropica 23: 242-254.

Ibarra-Manríquez G, Oyama K. 1992. Ecological correlates of reproductive traits of Mexican rain forest trees. Am. J. Bot. 79: 383-391.

Jaramillo V, Sanford R. 1995. Nutrient cycling in tropical deciduous forests. En: Mooney H, Medina E, Bullock S, edrs. Tropical deciduous forest ecosystems. Cambridge University Press, 346-361.

Jardel E. 1990. Conservación y uso sostenido de recursos forestales en ecosistemas de montaña. En: Rojas R, edr. En busca del equilibrio perdido: el uso de los recursos naturales en México, Universidad de Guadalajara, México, 209-235.

Jardel E, Sánchez-Velázquez LR, 1989. La sucesión forestal: Fundamento ecológico de la sivilcultura. Ciencia y Desarrollo 14 : 33-43.

Larson J, Eguiarte L. 1994. Demografía de Echeveria gibbiflora DC. (Crassulaceae) en dos ambientes contrstaantes del Pedregal de San Angel. En: Rojo A, compilador. Reserva ecológica «El Pedregal» de San Angel: ecología, historia natural y manejo. UNAM, México, 205-219.

León de la Cruz JL, Fanjul L. 1985. Movimiento estomático y estrategias adaptativas en diversas poblaciones de jojoba, Simmondsia chinensis (Link) Sch., en el norestes de México. Biótica 10: 7-17.

López-Portillo J, Ezcurra E. 1985. Phenology of Avicennia germinans L. in one year cycle in a mudflat at the Laguna de Mecoacán, Tabasco. Biotropica 17: 186-190.

López-Portillo J, Ezcurra E. 1989a. Mangrove responce to salinity in two geoforms. Funct. Ecol. 3: 355-361.

López-Portillo J, Ezcurra E. 1989b. Zonation in mangrove and saltmarsh vegetation in relation to soil characteristics and species interactions at the Laguna Mecoacan, Tabasco, Mexico. Biotropica 21: 107-114.

López-Portillo J, Ezcurra E, Maass J. 1989. Los Petenes de Sian Ka'an, Quintana Roo y su relación con gradientes de presión hídrica. Acta Bot. Mex. 5: 19-20.

Martínez ML, Rincón E. 1993. Growth analysis of Chamaechista chamaecristoides (Leguminosae) under contrasting nutrient conditions. Acta Oecol. 14: 521-528.

Martínez ML, Moreno-Casasola P, Rincon E. 1994. Sobrevivencia de una especie endémica de dunas costeras ante condiciones de sequía. Acta Bot. Mex. 26: 53-62.

Martínez-Ramos M. 1985. Claros, ciclos vitales de los árboles tropicales y la regeneración natural de las selvas altas perennifolias. En: Gómez-Pompa A, del Amo R S, edrs. Investigaciones sobre la regeneración de selvas altas en Veracruz, México Vol. II. Alhambra, México, 191-239.

Martínez-Ramos M. 1994. Regeneración natural y diversidad de especies arbóreas en selvas húmedas. Bol. Soc. Bot. México 54: 179-224.

Martínez-Ramos M, Soto-Castro A. 1993. Seed rain and advanced regeneration in a tropical rain forest. Vegetatio 108:299-318.

Martínez-Ramos M, Alvarez-Buylla E. 1986. Gap dynamics, seed dispersal and tree recruitment: the case of Cecropia obtusifolia at Los Tuxtlas, México. En: Estrada A, Fleming TH, edrs. Frugivores and seed dispersal. Dordrecht: Dr. Junk Publishers, 323-346.

Martínez-Ramos M, Sarukhán J, Piñero D. 1988a. The demography of trees in the context of forest gap dynamics: The case of Astrocaryum mexicanum at Los Tuxtlas tropical rain forest. En: Davy DJ, Hutchings MJ, Watkinson AR, edrs. Plant population ecology. Blackwell, Oxford, 293-313.

Martínez-Ramos M, Alvarez-Buylla E, Sarukhán J, Piñero D. 1988b. Treefall age determination and gap dynamics in a tropical rain forest. J. Ecol. 76: 700-716.

Martínez-Yrizar A, Sarukhán J. 1990. Litterfall patterns in a tropical deciduous forets in Mexico. J. Trop. Ecol. 6: 433-444.

Martínez-Yrizar A, Sarukhán J. 1993. Cambios estacionales del mantillo en el suelo de un bosque tropical caducifolio y subcaducifolio en Chamela, Jalisco, México. Acta Bot. Mex. 21: $1-6$.

Martínez-Yrizar A, Sarukhán J, Pérez-Jiménez A, Rincón E, Maass JM, Solís-Magallanes A, Cervantes L. 1992. Aboveground living phytomass of a tropical deciduous forest. J. Trop. Ecol. 8: 87-96.

Martínez-Yrizar A, Maass JM, Pérez-Jimenez LA, Sarukhán J, Rincón E. 1995. Net primary productivity of a tropical diciduous forest ecosystem in western Mexico.J. Trop. Ecol. (en prensa).

Maass JM, Jordan C, Sarukhán J. 1988. Soil erosion and nutrient losses in seasonal tropical agroecosystems under various management techniques. J. Appl. Ecol. 25: 595-607.

Maass JM, Vose JM, Swank WT, Martínez-Yrizar A. 1995. Seasonal changes of leaf area index (LAI) on a tropical deciduous forest in western Mexico. For. Ecol. Manag. (en prensa).

Maya Y, Arriaga L. 1995. Litterfall and phenological patterns of the dominant overstorey species of a desert scrub community. $J$. Arid Environ. (en prensa)

Meave del Castillo J, Soto MA, Calvo LM, Paz-Hernández H, Valencia S. 1992. Análisis sinecológico del bosque mesófilo de montaña de Omiltemi, Guerrero. Bol. Soc. Bot. México 52: 31-77.

Medina E, Mooney HA, Vázquez-Yanes C, edrs.1984. Physiological ecology of plants in the wet tropics. Dr. Junk Publishers, Dordrecht.

Mendoza A, Franco M. 1992. Integración clonal en una palma tropical. Bull. l'Inst. Francais d'est. Andines 21: 623-635.

Mendoza A, Piñero D, Sarukhán J. 1987. Effects of experimental defoliation on growth, reproduction and survival of Astrocaryum mexicanum. J. Ecol. 75: 545-554.

Miranda F, Hernández-X E. 1963. Los tipos de vegetación de México y su clasificación. Bol. Soc. Bot. México 29: 20-179.

Montaña C. 1990. A florsitic-structural gradient related to land forms in the sothern Chihuahan Desert. J, Veg. Sci. 1: 453-460. 
Montaña C, López-Portillo J, Mauchamp A. 1990. The response of two woody species to the conditions created by a shifting ecotone in an arid ecosystem. J. Ecol. 78: 789-798.

Montaña C, Ezcurra CE, Carrillo A, Delhome JP. 1988. The decomposition of litter in grasslands of northern Mexico: a comparasion between arid and non-arid environments. J. Arid Environ. 14: 55-61.

Moreno-Casasola P, Sánchez G. 1990. La enseñanza de la ecología en México. Ciencias (No. Especial) 4: 94-111.

Niño-Sulkowska MS, Lot A. 1983. Estudio demográfico del lirio acuático Eichornia crassipes (Mart.) Solms: dinámica de crecimeinto en dos localidades selectas de México. Bol. Soc. Bot. México 45: 71-83.

Núñez-Farfán J, Dirzo R. 1985. Herbivoría y sucesión en una selva alta perennifolia. En: Gómez-Pompa A, S. Del Amo S, edrs. Investigaciones sobre la regeneración de selvas en Veracruz, México. Alhambra, México, 313-332.

Núñez-Farfán J, Dirzo R. 1988. Within-gap spatial heterogeneity and seedling performance in a Mexican tropical forest. Oikos 51: 274-212.

Núñez-Farfán J, Dirzo R. 1991. Effects of defoliation on growth and survival of seedlings of a gap-colonizing neotropical tree. J.Veg. Sci. 2: 459-464.

Núñez-Farfán J, Dirzo R. 1994a. Evolutionary ecology of Datura stramonium $\mathrm{L}$. in central Mexico: natural selection for resistence to herbivorous insects. Evolution 48: 423-436.

Núñez Farfán J, Cabrales Vargas RA, González Astorga J. 1994. Estudios de ecología evolutiva en plantas herbáceas del pedregal de San Angel. En: Rojo A. Comp. Reserva ecológica "El Pedregal" de San Angel: ecología, historia natural y manejo. UNAM, México. 159-176.

Obieta MC, Sarukhán J. 1981. Estructura y composición de la vegetación herbácea de un bosque uniespecífico de Pinus hartwegii. I. Estructura y composición florística. Bol. Soc. Bot. México 41: 75-125.

Olmsted I, Alvarez-Buylla E. 1995. Sustainable harvesting of tropical trees: demography and matrix models of two palm species in Mexico. Ecol. Appl.

Oyama, K. 1990. Variation in growth and reproduction in the neotropical dioecious palm Chamaedorea tepejilote. J. Ecol. 78: 648-663.

Oyama, K. 1991. Seed predation by a curculionid beetle on the dioecious palm Chamaedorea tepejilote. Principes 35: 156160.

Oyama, K. 1993. Conservation biology of tropical trees demographic and genetic considerations. Environ. Update 1: 17-32.

Oyama K, Dirzo R. 1988. Biomass allocation in the dioecious palm Chamaedorea tepejilote and its life history consequences. Pl. Species Biol. 3: 27-33.

Oyama K, Dirzo R.1991. Ecological aspects of the interaction between Chamaedore tepejilote and Calyptocephala marginipennis, a herbivore bettle, in a Mexican rain forest. Principes 35: 86-93.

Oyama K, Mendoza A. 1990. Effects of defoliation on growth, reproduction, and survival of a neotropical dioecious palm, Chamadorea tepejilote. Biotropica 22: 119-123.

Palomeque R. 1988. Demografía y herbivoría en Omphalea oleifera (Euphorbiaceae). Tesis de licenciatura. UNAM, México.

Pérez-Nasser N, Vázquez-Yanes C. 1986. Longevity of buried seed from some tropical rain forest trees and shrubs of Veracruz, Mexico. Malay. For. 49: 352-356.

Pérez-Nasser N, Eguiarte L, Piñero D. 1993. Mating systems and genetic structure of the distylous tropical tree Psychotria faxlucens Lorence \& Dwyer (Rubiaceae). Am. J. Bot. 80: 45-52.

Piñero D, Eguiarte L. 1987. The origin and biosystematic status of
Phaseolus coccineus: electrophoretic evidence. Euphytica 37: 199-203.

Piñero D, Sarukhán J. 1982. Reproductive behaviour and its individual variability in a tropical palm, Astrocaryum mexicanum. J. Ecol. 70: 461-472.

Piñero D, Sarukhán J, Alberdi P. 1982. Reproductive behaviour and its individual variability in a tropical palm, Astrocaryım mexicanum. J. Ecol. 70: 461-472.

Piñero D, Martínez-Ramos M, Sarukhán J. 1984. A population model of Astrocaryum mexicanum and a sensitivity analysis of its finite rate of increase. J. Ecol. 72: 977-991.

Piñero D, Sarukhán J, González E. 1977. Estudios demográficos en plantas. Astrocaryum mexicanum Liebm. I. Estructura de las poblaciones. Bol. Soc. Bot. México 37: 69-118.

Popma J, Bongers F, Martínez-Ramos M, Veneklaas E. 1988. Pioneer species distribution in treefall gaps in neotropical rainforest: a gap definition and its consequences. J. Trop. Ecol. 4: 77-88.

Puig HR, Bracho, Sosa V.1983. Compisción florística y estructura del bosque mesófilo de montaña en Gómez-Farías, Tamaulipas, México. Biótica 8: 339-359.

Purata S. 1986. Floristic and structural changes during old-field succession in the Mexican tropics in relation to site history and species availability. J. Trop. Ecol. 2: 257-276.

Quiroz A. 1981. Nota sobre la clasificación de hidrófitas en relación a la química del agua. Biótica 6: 1-5.

Rico V. 1989. The importance of floral and circumfloral nectar to ants inhabiting dry tropical lowlands. Biol. J. Linn. Soc. 38: 173181.

Rincón E, Huante P. 1993. Growth responses of tropical deciduous tree seedlings to contrasting light conditions. Tree Struc. Funct. 7: 202-207.

Rincón E, Huante P. 1994. Influence of mineral nutrient availability on growth of tree seedlings from the tropical deciduos forest. Tree Struc. Funct. 9: 93-97.

Rodríguez Hernández C, Vásquez Yáñez C. 1992. La conservación de plantas en peligro de extinción a través del almacenamiento a largo plazo de semillas. Interciencia 17: 293-297.

Rojo A (Compilador). 1994. Reserva ecológica «El Pedregal» de San Angel: ecología, historia natural y manejo. UNAM, México.

Rzedowski J. 1978. Vegetación de México. Limusa, México.

Rzedowski J. 1991. Diversidad y origenes de la flora fanerogámica de México. Acta Bot. Mex. 14: 3-21.

Sánchez-Coronado M, Rincón E, Vázquez-Yanes C. 1990. Growth responses of three Piper especies growing under constrating light conditions. Can. J. Bot. 68: 1182-1186.

Sarukhán J. 1964. Estudio sucesional de un área talada en Tuxtepec, Oax. Pub. Esp. Inst. Nal. Inv. For, México 3: 67-172.

Sarukhán J. 1968. Análisis sinecológico de la selva de Terminalia amazonica en la planicie costera del Golfo de México. Tesis de Maestría. Colegio de Postgraduados, ENA, Chapingo, México.

Sarukhán J. 1978. Studies on the demography of tropical trees. En: Tomlinson PB, Zimmerman MH, edrs. Tropical trees as living systems. Cambridge University Press, London, 163-184.

Sarukhán J. 1980. Demographic problems in a tropical system. En: Solbrig OT, edr.Demography and evolution in plant populations. University of California Press, Berkeley, 168-188.

Sarukhán, J. 1981. México en: Kormondy, EJ, McCormick JF, edrs. Handbook of contemporary developments in word ecology. Greewood Press, Westport, CT. 35-51.

Sarukhán J, Harper JL. 1973. Studies on plant demography. Ranunculus repens. $R$. acris y $R$. bulbosus I. Population flux and survivosrhip. J. Ecol. 61: 675-716. 
Sarukhán J, Maass JM. 1990. Bases ecológicas para el manejo sostenido de ecosistemas: el sistema de cuencas hidrológicas. En: Leff E, edr. Medio ambiente y desarrollo en México Vol 1. UNAM (CIIH)-Porrúa, México, 81-114.

Sarukhán J, Martínez-Ramos M, Piñero D. 1984. The analysis of demographic variability at the individual level and its population consequences. En:Dirzo R, Sarukhán J, edrs. Perspectives on plant population ecology. Sinauer Sunderland, Massachusetts, 141-165.

Sarukhán J, Piñero D, Martínez-Ramos M. 1985. Plant demography: a community level interpretation. En: White J, edr. Studies in plant demography: a festschrift for John L. Harper. Academic Press, London, 17-31.

Ting IP, Lord EM, Sternberg L, Deniro J. 1985. Crassulaceae acid metabolism in the strangler Clussia rosea. Jacq. Science 229: 969-971.

Tinoco-Ojanguren R, Pearcy W. 1992. Dynamic stomatal behavior and its role in carbon gain during sunflecks of a gap phase and an understory Piper species acclimated to high and low light. Oecologia 92: 222-228.

Tinoco-Ojanguren YR, Pearcy W. 1993a. Stomatal dynamics and its importance to carbon gain in the two rainforest Piper species. I. VPD effects on the transient stomatal responce to lightflecks. Oecologia 94: 388-394.

Tinoco-Ojanguren C, Pearcy RW. 1993b. Stomatal dynamics and its importance to carbon gain in the two rainforest Piper species. II. Stomatal versus biochemical limitations during photosynthetic induction. Oecologia 94: 395-402

Tinoco-Ojanguren C, Vázquez-Yanes C. 1983. Especies CAM en la selva húmeda tropical de Los Tuxtlas, Veracruz. Bol. Soc. Bot. México 45: 150-153.

Tinoco-Ojanguren C, Vázquez-Yanes C. 1983. Diferencias en poblaciones de Piper hispidum bajo condiciones de luz contrastantes en una selva alta perennifolia. Biótica 8: 281-293.

Toledo V.1992. What is ethnoecology?: Origins, scope and implications of a rising discipline. Etnoecológica 1: 5-22.

Valdés AM, Piñero D. 1992. Phylogenetic estimation of plasmid exchange in bacteria. Evolution 46: 641-656.

Valiente A, Ezcurra E. 1991. Shade as a cause of the association between the cactus Neobuxbaumia tetetzo and the nurse Mimosa luisiana. J. Veg. Sci. 2: 11-14.
Valiente A, Vite F, Zavala-Hurtado A. 1991a. Interaction between the cactus Neobuxbaunia tetezo and the nurse shrub Mimosa luisiana. J. Veg. Sci. 2: 11-14.

Valiente A, Bolongaro-Crevera A, Briones O, Ezcurra E., Rosas M, Núñez H, Barnard G, Vázquez E. 1991b. Spatial relationship between cacti and nurse srhubs in a semi-arid environment in central Mexico. J. Veg. Sci. 2: 15-20.

Vázquez-Yanes C. 1980. Notas sobre la autoecología de los árboles pioneros de rápido crecimiento de la selva tropical lluviosa. Trop. Ecol. 21: 103-112.

Vázquez-Yanes C. 1995. Una historia de investigación científica en plantas estranguladoras. Ciencias 37: 57-64.

Vázquez-Yanes C, Orozco-Segovia A. 1984. Ecophysiology of seed germination in tropical humid forest of the word: a review. En: Medina E, Mooney HA, Vázquez-Yanes C, edrs. Physiological ecology of plants in the wet tropics. Dr. Junk Publishers, Dordrecht, 37-49.

Vázquez-Yanes C, Orozco-Segovia A. 1993. Patterns of seed longevity and germination in the tropical rainforest. Ann. Rev. Ecol. Syst. 24: 69-87.

Vázquez-Yanes C, Orozco-Segovia A, Rincón E, Sánchez-Coronado ME, Huante P, Barradas V, Toledo JR. 1990. Light beneath the litter in a tropical forest: effect on seed germination. Ecology 71: 1952-1958.

Venable L. 1984. Using intraspecific variation to study the ecological significance and evolution of plant life-histories. En: Dirzo R. Sarukhán J, edrs.Perspectives on plant population ecology. Sinauer Sunderland, Massachusetts, 166-187.

Vite F, Portilla E, Zavala JA, Valverde PL, Díaz A. 1995. A natural hybrid population between Neubouxbaumia tetezo and Cephalocereus columna-tejanii (Cactaceae). J. Arid Environ. (en prensa).

Wendt T. 1991. Composition, floristic affinities, and origins of the canopy tree flora of the Mexican Atlantic slope rain forests. En: Ramamoorthy TP, Bye R, Lot A, edrs. Biological diversity of Mexico: origins and distribution. Oxford University Press, New York, 595-680.

White J, edr. 1985. Studies in plant demography: a festschrift for John L. Harper. Academic Press, London.

Wilson EO. 1992. The diversity of life. Harvard University Press.

Zavala A, Díaz A. 1995. Repair, growth, age and reproduction in the giant columnar cactus Cephalocereus hoppenstedtii K. Sch. (Cactaceae). J. Arid Environ. (en prensa). 